\title{
Commentary: What's in an algorithm?
}

\author{
Joshua S. Wolovits, MD, ${ }^{\mathrm{a}, \mathrm{b}}$ and Camille L. Hancock Friesen, MD ${ }^{\mathrm{b}, \mathrm{c}}$
}

\footnotetext{
From the a Division of Critical Care, Department of Pediatrics, University of Texas Southwestern Medical Center, Dallas, Tex; ${ }^{\mathrm{c}}$ Division of Pediatric Cardiothoracic Surgery, Department of Cardiovascular and Thoracic Surgery, University of Texas Southwestern Medical Center, Dallas, Tex; and ${ }^{\mathrm{b}}$ Children's Medical Center, Dallas, Tex.

Disclosures: Authors have nothing to disclose with regard to commercial support.

Received for publication April 16, 2019; accepted for publication April 16, 2019; available ahead of print May 31, 2019.

Address for reprints: Camille L. Hancock Friesen, MD, 1935 Medical District Dr, Dallas, TX 75235 (E-mail: Camille.HancockFriesen@UTSouthwestern.edu).

J Thorac Cardiovasc Surg 2019;158:1218-9

0022-5223/\$0.00

Published by Elsevier Inc. on behalf of The American Association for Thoracic Surgery https://doi.org/10.1016/j.jtcvs.2019.04.062
}

The "triple aim" as articulated by the Institute for Healthcare Improvement includes improving population health, improving patient experience, and reducing health care costs. ${ }^{1}$ Although tackling this is no small undertaking, reducing variability in practice is one strategy used to achieve this set of aims. ${ }^{2}$ In this issue of the Journal, Bertrandt and colleagues ${ }^{3}$ present a well-designed and wellexecuted quality improvement project aimed at reducing variation in a common postsurgical practice: chest tube management and removal. Although seemingly mundane, the effect of reducing variation and optimizing timing of chest tube removal has never been assessed. Does more timely chest tube removal improve patient experience and reduce health care costs?

In 126 patients with 277 chest tubes in the course of 20 months, Bertrandt and colleagues ${ }^{3}$ instituted a process aimed at reducing chest tube duration by (1) standardizing removal criteria on the basis of historical mean volumes of output preceding chest removal and (2) performing twice daily assessments of chest tube removal "readiness." The results of this simple practice change are impressive, with a reduction in the average chest tube duration by 14 hours (61 hours before implementation vs 47 hours after implementation). The $22 \%$ reduction in time to chest tube removal was associated with significant reductions in hospital length of stay and costs. ${ }^{3}$ The rate of chest tube reinsertion, a balancing measure, was unchanged. This lack of change supports the contention that there was no incremental risk associated with earlier removal. Although this reduction in time to chest tube removal is admirable, the low reinsertion rate opens up the question of whether this strategy could be even more "aggressive."

Bertrandt and colleagues ${ }^{3}$ used the electronic health record to implement this initiative by developing a "chest tube rounding navigator" tool that prompts assessment of readiness for removal and documentation of chest tube status. This process is instructive not just for providing a road map for replicating early chest tube removal in other radiography? unknown.

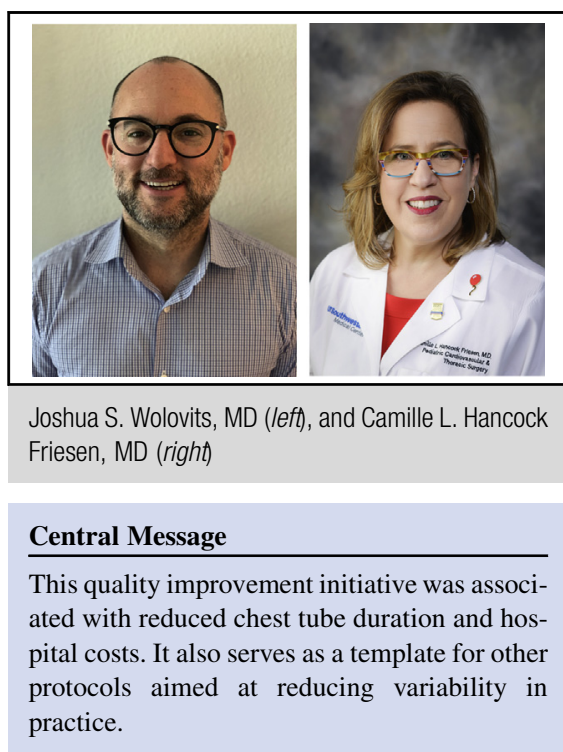

See Article page 1209.

institutions but for informing other quality initiatives aimed at reducing practice variation. Might a similar strategy be applied to other common practices with possibly similar positive effects, such as routine blood draws and

The applicability of this algorithm is not universal. Exclusions including chylothorax, high-volume drainage, neonates, and univentricular repairs resulted in the inclusion of only 126 patients in 20 months, with an average STAT category of only 1.7. Although we certainly agree with the initial implementation of such an algorithm for the "healthiest" patients requiring cardiac surgery, its applicability and impact in more complex cardiac patients is still

The reduction in time to chest tube removal was associated with significant reductions in hospital length of stay and cost, but the direct effect on these outcomes should be taken with pause. Although earlier chest tube removal may improve patient experience, it is not intuitively obvious how an average 14 hour reduction in time to removal is associated with a nearly 3-day reduction in length of stay. The relationship between these variables is likely nuanced and potentially confounded by concomitant hospital-wide initiatives aimed at reducing length of stay and costs. In addition, although the results presented suggest wide adoption of the practice change, noted by narrowed confidence 
limits, the sustainability of this practice change is not addressed in this report.

With this algorithm, Bertrandt and colleagues ${ }^{3}$ have enhanced quality and value through a structured systemslevel approach to a universal element of pediatric cardiac surgery, namely chest tube management. Clearly more than just an algorithm, this is an example of optimal delivery of modern health care.

\section{References}

1. Berwick DM, Nolan TW, Whittington J. The triple aim: care, health and cost Health Aff (Millwood). 2008;27:759-69.

2. Farias M, Jenkins K, Lock J, Rathod R, Newburger J, Bates D, et al. Standardized clinical assessment and management plans (SCAMPs) provide a better alternative to clinical practice guidelines. Health Aff (Millwood). 2013;32:911-20.

3. Bertrandt RA, Saudek DM, Scott JP, Madrzak M, Miranda MB, Ghanayem NS et al. Chest tube removal algorithm is associated with decreased chest tube duration in pediatric cardiac surgical patients. J Thorac Cardiovasc Surg. 2019;158: 1209-17. 